\title{
Research on improved strategy of ant colony optimization algorithm
}

\author{
Wang Jinguo ${ }^{1, a}$, Wang $\mathrm{Na}^{2, b^{*}}$ (corresponding author),Ma Haichun ${ }^{3, \mathrm{c}}$ \\ ${ }^{1}$ Department of Urology, the First Hospital of Jilin University,China \\ ${ }^{2}$ Department of Anesthesiology, the First Hospital of Jilin University, China \\ ${ }^{3}$ Department of Anesthesiology, the First Hospital of Jilin University, China \\ a'wangjinguolily@163.com, ${ }^{\mathrm{b}}$ lilyly12345@163.com, ${ }^{\mathrm{C} X i a o b e n 6666 @ 126 . c o m ~}$
}

Keywords: ant colony algorithm. Pheromone update.

Abstract. This paper summarizes three kinds of improved strategies for ant colony optimization algorithm: the improved strategy based on the structure of solution, the improved strategy based on the pheromone updating, and the improved method based on the solution processing. Taking the ant colony system as an example, this paper expounds the three improved strategies and the improvement effect of these strategies are verified and compared by experiments.

\section{Introduction}

Ant colony optimization algorithm is inspired by the research of real ant colony behavior. Biology research showed a mutual cooperation of ants can find the shortest route between the source of food and the nest, and one ant is not[1]. After a lot of observation, biologists found that the ant will leave a pheromone substances in the path of its movement. The pheromone will help ant transmission the information, and the ants can perceive the material concentration in the movement. The higher the pheromone concentration, the greater the probability that the ants choose, so the pheromone concentration will be further enhanced[2]. Ant colony collective behavior, therefore, showed a positive feedback of information: the more ants, the more probability of the route will be chosen. Through the indirect communication mechanism between individual, the ants can get the shortest route for search food. Since the ant system proposed, many improved ant system has been proposed and successfully applied to many optimization problems[3].

\section{Improved strategy of ant colony optimization algorithm}

Improved strategy based on the structure of solution. The structure of the solution is an important for the ant system and other ant colony optimization algorithms [4]. Excellent construction method can improve the performance of the algorithm. The improvement of the solution structure is mainly concentrated in the following two aspects:

Use a candidate list. The candidate list is a special data structure associated with the solution elements. A candidate list is a property of a solution element, and each element has a candidate list. Moreover, the candidate list of solution stores other solution elements but own. The solution elements in the candidate list are ranked according to some heuristic criteria and rank from high to low on the candidate list. The candidate list is calculated before the searching process of algorithm, and remains unchanged during the operation of the algorithm.

Change transfer rule. Some ant colony optimization algorithm has changed the transfer rules [5]. The artificial ant uses a pseudo random proportional probability model as the transfer rule in the ant colony system. The model introduced an additional parameter $\mathrm{q}_{0}$ and a random variable $\mathrm{q}$. They are both belong to $[0,1][6]$. For the ant colony system, the probability that the artificial ant $\mathrm{K}$ in the city 
$\mathrm{i}$ choose the next city $\mathrm{j}$ is:

If $q \leq q_{0}$

$p_{i j}^{k}(t)=\left\{\begin{array}{l}1 \quad \text { if } j=\arg \max _{j \notin t a b u_{k}} \tau_{i j}(t) \cdot \eta_{i j}^{\beta} \\ 0 \quad \text { otherwise }\end{array}\right.$

Else

$$
p_{i j}^{k}(t)= \begin{cases}\frac{\tau_{i j}(t) \cdot \eta_{i j}^{\beta}}{\sum_{l \notin t a b u_{k}} \tau_{i l}(t)^{\alpha} \cdot \eta_{i l}^{\beta}} & j \notin t a b u_{k} \\ 0 & \text { otherwise }\end{cases}
$$

The transfer rule of the ant colony system is essentially the greedy selection that make full use of the known information and heuristic information with the probability $\mathrm{q}_{0}$. It choose the city that meet the constraints and make the $\tau_{i j}(t) \cdot \eta_{i j}^{\beta}$ maximum as the direction of the artificial ants movement.

Improved strategy based on pheromone update. The pheromone update can affect the convergence speed of the algorithm, the average quality of the algorithm solution and the quality of the optimal solution. Pheromone updating is the key to improve the ant colony optimization algorithm. Ant colony system in addition to the local pheromone update, but also for global pheromone update. In ant colony system, the global pheromone update is performed only on the edge of the global optimal solution. Global pheromone update rules are as follows:

$\tau_{i j}(t+1)=\left\{\begin{array}{cc}(1-\rho) \cdot \tau_{i j}(t)+\rho \cdot \Delta \tau_{i j}^{\prime}(t) & (i, j) \in e(t) \\ \Delta \tau_{i j}(t) & \text { otherwise }\end{array}\right.$

In which

$\Delta \tau_{i j}^{\prime}(t)=\left\{\begin{array}{cc}\frac{1}{f(e(t))} & (i, j) \in e(t) \\ 0 & \text { otherwise }\end{array}\right.$

From the local and global pheromone updating rules of ant colony system, the edge of the artificial ants passed on the pheromone concentration decreases, and contain the global optimal solution will increase, others, remain unchanged.

Improved method based on the solution processing. The premise of the solution processing is that we know some feasible solutions to the problem. In each iteration of the ant colony optimization algorithm, each artificial ant can construct a complete and feasible solution, which provides the possibility for the solution processing. Local search is a kind of efficient technology improvement to the solutions, commonly used to solve difficult combinatorial optimization problem. It's also an important method to improve the performance of ant colony optimization algorithm which is integrated in the ant colony optimization algorithm for solving combinatorial optimization problems.

In general, local search can improve the quality of the solution in a reasonable time. The effect that combined local search and colony optimization is more obvious than respectively. Local search is needed from a feasible solution. Because there has correlation between the solution quality and the distance with optimal solution, a good initial solution is particularly important for local search. Ant colony optimization algorithm can provide a large number of high quality initial solutions for local search. But it needs to be explained that the local search is usually found the local optimal 
solution within the search space rather than the optimal solution of problem.

\section{Experiment and result analysis}

In this paper, we use TSP to test the improvement algorithm. Experiment selects the ST70 problem instance. The parameters in the algorithm are as follows:

$$
m=100, Q=100, \alpha=0.8, \beta=0.7, \rho=0.9, q_{0}=0.9
$$

The number of simulation experiments is 5. Each time is the comparison the improved algorithm and the original algorithm. Test results are as follows:

Table 1.Comparison of ant colony optimization algorithm

\begin{tabular}{|c|c|c|}
\hline Number & Original algorithm & Improved algorithm \\
\hline 1 & 708.5 & 690.8 \\
\hline 2 & 683.4 & 678.9 \\
\hline 3 & 697.2 & 683.5 \\
\hline 4 & 677.8 & 670.9 \\
\hline 5 & 689.4 & 680.1 \\
\hline 2800 \\
2600 \\
2400 \\
2200 \\
2000 \\
1800 \\
1600 \\
1400 \\
1200
\end{tabular}

Fig1. The convergence figure of the improved ant colony algorithm

As can be seen from the table 1, the optimal solution obtained by the original ant colony algorithm is 677.8 , while the worst solution is 708.5. But the improved algorithm can get the optimal solution is 670.9 , the worst solution can be 690.8 . From figure 1 , we can see the improved algorithm has fast convergence, and is not easy to enter into the premature stagnation state to search out the path more excellent.So we can see that the improved algorithm can produce a optimal path, and has better effect. 


\section{Conclusion}

In this chapter, an improved ant colony algorithm is proposed to solve the shortcomings of the basic ant colony algorithm, which is easy to fall into local optimal solution. There has three aspects improvement: the parameters initialization, the probability of the state transition, and pheromone update. Experimental results that the improved ant colony algorithm can speed up the convergence of the algorithm by changing the pheromone. In the same number of iterations, the path is greatly shortened.

\section{References}

[1] Dorigo M,Gambardella L M.Ant colony system: A cooperative learning approach to the traveling salesman problem. IEEE Transactions on Evolutionary Computation . 1997.

[2] WHITET,etal. Connection management using adaptive mobile agents. In Proceedings of the International Conference on Parallel and Distributed Processing Techniques and Applications . 1998.

[3] DICAROG,DORIGOM. Extending Ant Net for best-effort Quality-of-Service routing. Proc. ANTS '98-First International Workshop on Ant Colony Optimization . 1998.

[4] J.G. Taylor, "Neural computation: The historical background," in E. Fiesler and R. Beale, eds., Handbook of Neural Computation, New York: Oxford University Press, 1997.

[5] Xiang-Sun Zhang, Neural Networks in optimization, volume46 of Nonconvex Optimizations, 2002.

[6] Tan KC,Lee H L,Q.L.Zhu, OuK, Heuristic methods for vehicle routing Problems with time windows[J]. Artificial intelligence in Engineering,2001,15(3):281 - 295. 